\title{
Lung Segmentation Using Proposed Deep Learning Architecture
}

\author{
https://doi.org/10.3991/ijoe.v16i15.17115 \\ Hayder Ayad \\ Al-Bayan University, Baghdad, Iraq \\ Ikhlas Watan Ghindawi ( $\left({ }^{\prime}\right)$ \\ Al-Mustansiriyah University, Baghdad, Iraq \\ ikhlas wataneuomustansiriyah.edu.iq \\ Mustafa S. Kadhm \\ Imam Ja'afar Al-Sadiq University, Baghdad, Iraq
}

\begin{abstract}
The Prediction and detection disease in human lungs are a very critical operation. It depends on an efficient view of the CT images to the doctors. It depends on an efficient view of the CT images to the doctors. The clear view of the images to clearly identify the disease depends on the segmentation that may save people lives. Therefore, an accurate lung segmentation system from CT image based on proposed CNN architecture is proposed. The system used weighted Softmax function the improved the segmentation accuracy. By experiments, the system achieved a high segmentation accuracy $98.9 \%$ using LIDCIDRI CT lung images database.
\end{abstract}

Keywords - CT images, lung segmentation, DNN, CNN, Softmax

\section{Introduction}

Lung Segmentation in CT images is a very important and useful technique in medical field [1]. An efficient and accurate segmentation results lead for better disease prediction and detection. Now days with the pandemic of COVID-19 the needs of reliable and accurate system of segmenting the lung became more importance.

DNN is successfully applied in segmentation of the medical images $[2,3]$ and other recognition and detection systems $[4,5,6]$. Several deep networks with various activation functions are obtained a satisfied-results in different researches and existing systems [7], [8]. Therefore, we will use the CNN in our proposed work for better segmentation results.

The most recent researches related to the lung segmentation system Qinhua Hu et al. [9] used the Convolutional Neural Network (CNN) Mask R-CNN with the K-means kernel for segmenting the lung from $\mathrm{CT}$ images. The authors mentioned that their system achieved a high segmentation accuracy $97.68 \%$ and low runtime 11.2 seconds. 
Sarah E. et al. [10] present deep learning framework for lung segmentation with Acute Respiratory Distress Syndrome (ARDS) using novel multi-resolution convolutional neural network (ConvNet). The authors used Transfer learning to accommodate the limited number of training datasets. The obtained results were $91-96 \%$ using multiresolution model.

Brahim A. et al. [11] employ the U-net architecture of deep learning for segmenting the lung. The architecture extract high-level information and a symmetric expanding path which is recover the needed information based on the contracting path. Based on end-to-end training, the used architecture gives $95 \%$ accuracy using only very few images.

Yuchong Gu. Et al. [12] investigated deep convolutional neural network (DCNN) for lung area segmentation based on U-Net and V-Net. The both networks are used as a baseline networks and a new multi-scale prediction network (MPN) is designed for better dice coefficient results. The system results were $87-98 \%$ using MPM, 93-98\% using U-Net and 52-97\% using V-Net.

Tao P. et al. [13] present a system for lung Region of Interest (ROI) boundaries detection. Hull-Closed Polygonal Line Method (Hull-CPLM) is used for detecting the ROI in chest radiographs based on two steps which are preprocessing and refinement. Besides, the system performance was $97.08 \%$ using Dice Similarity Coefficient (DSC) evaluation and public lungs database.

\section{Proposed System}

A proposed $\mathrm{CNN}$ architecture is employed for segmenting the input lung from CT image. First the system used a standard CT lung images database called LIDC-IDRI [14]. The proposed CNNs architecture is illustrated in figure 1.

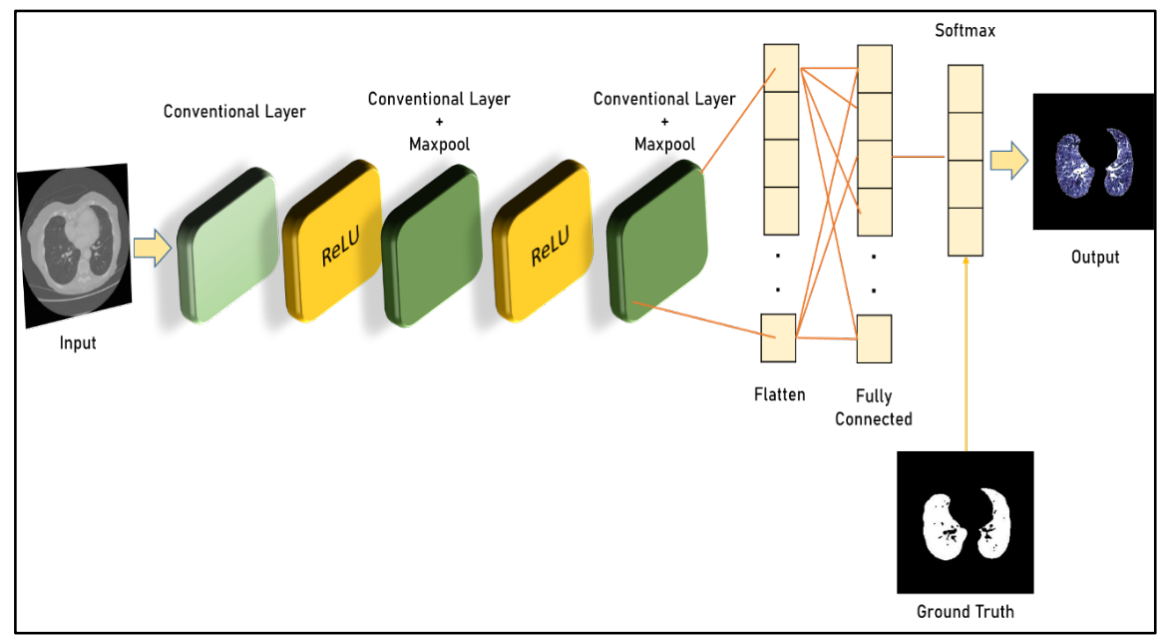

Fig. 1. Proposed CNN architecture 
Proposed CNN architecture is first trained by the input CT images and their corresponding masks. However, corresponding mask is generated for the input image as an output during the testing stage. After that, the generated mask is used to segment the required are of the input CT image. The last step of the proposed system is the use of Softmax function. In our system a weighted Softmax function is used to match the output segmented image within the ground truth image for accurate segmentation result. Softmax could be interpreted using equation 1:

$$
S p=\frac{1}{M} \sum_{j=0}^{n} \sum_{i=0}^{n}-\log \operatorname{Pr}\left(A=a_{i, j} / \mathrm{B}=b_{i, j}\right)
$$

Where,

$S p \quad=$ The Probability of Softmax function

$a_{i, j} \quad=$ The average of weight

$b_{i, j}=$ The pixel of the input image

$i, j \quad=$ The iteration of the value

$\operatorname{Pr}=\mathrm{A}$ and $\mathrm{B}$ probability function

The probability function under the Softmax function is the main focus of the proposed system. In equation 1 a bias probability is included, in order to push the strong biases in the input data away. The data could be normalized by adding a positive weight to a high negative bias and adding a negative weight to a high positive bias.

The bias probability element is increased, when the value of the weight is very small, due to a very small denominator. Proposing a new weight called Extra Weight (w1) can enhance the equation 2 :

$$
M y_{i, j}=\sum_{j=0}^{j=m} \sum_{i=0}^{m} M W_{i, j} x_{i, j}+\frac{e^{M W_{i, j}}}{\sum_{i, j} e^{E W_{1}+M W_{i, j}}}
$$

Where,

$$
\begin{aligned}
& M y_{i, j}=\quad \text { The improved function of evidence } \\
& M W_{i, j}=\quad \text { The main weight } \\
& \mathrm{EW}_{1}=\text { Extra Weight } \\
& \boldsymbol{e}^{\boldsymbol{E} \boldsymbol{W}_{\boldsymbol{i}, j}}=\mathbf{T} \text { The Weighted Exponential }
\end{aligned}
$$

$i, j=$ The iteration of the value

By applying the equation 1 will normalize the evidence function will make the system obtain a better segmentation accuracy.

\section{Results and Dissection}

In the proposed system Python 3.8.3 programing language is used with Tensor Flow 2.2 library based on Keras for implementing the proposed system. Besides, NVIDIA GeForce with 640 CUDA Cores and 8 GB of memory in order to exploit its computational speed for better and authenticate results. The used network parameters were:

- Batch size: 64 .

- Number of epochs: 70 . 
In the proposed work the configurations of the CNN shown in table 1. The depth of the configurations increases from the left (A1) to the right (A4), as more number of layers are added. The convolutional layer parameters are denoted as conv with its number in the table.

Table 1. Configurations of the proposed CNN architecture

\begin{tabular}{|l|l|l|l|}
\hline \multicolumn{1}{|c|}{ A1 } & \multicolumn{1}{c|}{ A2 } & \multicolumn{1}{c|}{ A3 } & \multicolumn{1}{c|}{ A4 } \\
\hline 10 weighted layers & 10 weighted layers & 15 weighted layers & 15 weighted layers \\
\hline Input CT Lung image & $\begin{array}{l}\text { Conv64 } \\
\text { ReLU }\end{array}$ & Conv64 & Conv64 \\
\hline Conv64 & Conv128 & Conv128 \\
\hline Maxpool1 & Conv128 & Conv256 \\
\hline Conv128 & Conv256 & Conv256 \\
\hline Maxpool2 & Conv512 \\
\hline Conv256 & Conv512 \\
\hline Maxpool3 & \\
\hline Conv512 & \\
\hline Maxpool4 & \\
\hline FC1 & FC2 \\
\hline Softmax
\end{tabular}

Furthermore, 512 CT images of LIDC-IDRI database are used to evaluate the proposed system. Figure 2 shows a samples images of the used database.

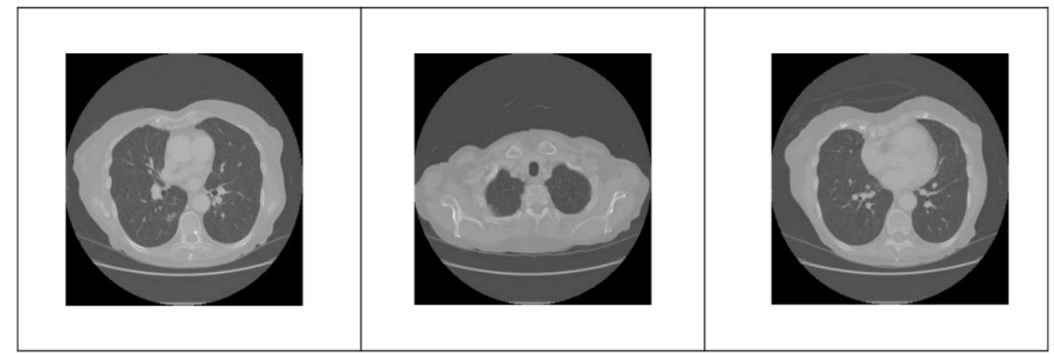

Fig. 2. The LIDC-IDRI CT lung sample images

A result of applying the proposed $\mathrm{CNN}$ architecture on three different images is illustrated in figure 3 . 


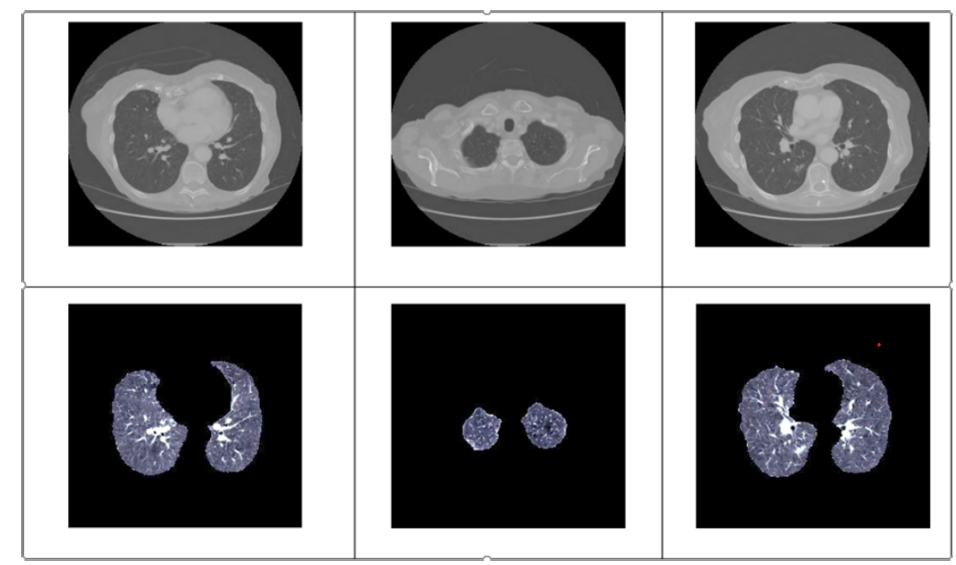

Fig. 3. The Segmentation results of the proposed CNN architecture

In addition, dice coefficient index DSC is used for evaluating the proposed network performance. DSC is obtained using the equation 3 below:

$$
D S C=2 * \frac{\|S \cap T\|}{\|S \oplus T\|}
$$

The obtained lung area presented by $\mathrm{S}$, in the proposed approach, and the ground truth image presented by T. By applying the equation 6 the system achieved $98.9 \%$.

In another hand, using ReLU activation function and the modified Softmax function leads the proposed system accurate result. Table 2 shows the effect of use the standard Softmax function and the modified one.

Table 2. Comparison result of proposed method and standard Softmax function

\begin{tabular}{|l|c|}
\hline \multicolumn{1}{|c|}{ Method } & Accuracy\% \\
\hline Softmax & 96.2 \\
\hline Proposed & 98.9 \\
\hline
\end{tabular}

\section{Conclusion}

An accurate lung segmentation system is presented in this paper. The system used proposed deep learning architecture based on CNN, ReLU activation function, and modified Softmax function. The system achieved a high segmentation result which is 98.9\% using LIDC-IDRI database. In the future work, the proposed system could be employed for detecting the COVID-19 in the CT lung images of the infected patients. Several DNN could be used for achieving high detection accuracy with lowest detection errors. 


\section{$5 \quad$ References}

[1] Ferlay J, Soerjomataram I, Dikshit R, Eser S, Mathers C, Rebelo M, et al. Cancer incidence and mortality worldwide: sources, methods and major patterns in GLOBOCAN 2012. Int J Cancer 2015; 136: E359-86. https://doi.org/10.1002/ijc.29210

[2] Bhavanishankar K, Sudhamani MV. Techniques for detection of solitary pulmonary nodules in human lung and their classifications -a Survey. Int J Cybern Inf 2015; 4:27-40.

[3] Kadhm, M.S. and Hassan, A.K.A., Handwriting word recognition based on SVM classifier. International Journal of Advanced Computer Science \& Applications, 1, pp.64-68, 2015.

[4] Liwei Geng, Tingting Dong, An Agricultural Monitoring System Based on Wireless Sensor and Depth Learning Algorithm, International Journal of Online and Biomedical Engineering (iJOE) - Vol. 13, No. 12, pp. 127-137, 2017. https://doi.org/10.3991/ijoe. v13i12.7885

[5] Ahmed Elnakib, Hanan M. Amer, Early Lung Cancer Detection Using Deep Learning Optimization, International Journal of Online and Biomedical Engineering (iJOE), Vol 16, No 06, pp. 82-94, 2020. https://doi.org/10.3991/ijoe.v16i06.13657

[6] Yaser Saleh, Ghassan F. Issa, Arabic Sign Language Recognition through Deep Neural Networks Fine-Tuning, International Journal of Online and Biomedical Engineering (iJOE), Vol. 16, No. 5, pp. 71-83, 2020. https://doi.org/10.3991/ijoe.v16i05. $\underline{13087}$

[7] Shaymaa A., Abdulrahman Wael Khalifa, Mohamed Roushdy, Mohamed Roushdy, AbdelBadeeh, Comparative study for 8 computational intelligence algorithms for human identification, Computer Science Review, Elsevier, Volume 36, pp.1-9, 2020. https://doi.org/10.1016/j.cosrev.2020.100237

[8] Hassan, A.K.A. and Kadhm, M.S., an Efficient Preprocessing Framework for Arabic Handwriting Recognition System. Diyala Journal for Pure Science, 12(3-part 2), pp.147-163, 2016.

[9] Qinhua Hua, Luís Fabrício de F. Souzab, Gabriel Bandeira Holandab, Shara S. A. Alvesc, Francisco Hércules dos S. Silvab, Tao Hand, Pedro P. Rebouças Filhob, An Effective Approach for CT Lung Segmentation using Mask Region-based Convolutional Neural Networks, Artificial Intelligence in Medicine, Elsevier, Volume 103, March 2020, 1017922020. https://doi.org/10.1016/j.artmed.2020.101792

[10] Sarah E. Gerarda, Jacob Herrmanna,c, David W. Kaczkaa,b,c, Guido Muschd, Ana Fernandez-Bustamantee, Joseph M. Reinhardt, Multi-Resolution Convolutional Neural Networks for Fully Automated Segmentation of Acutely Injured Lungs in Multiple Species, Medical Image Analysis, Elsevier, Volume 60, February 2020, 101592. https://doi.org/10.1016/j.media.2019.101592

[11] Brahim AIT SKOURT, Abdelhamid EL HASSANI, Aicha MAJDA, Lung CT Image Segmentation Using Deep Neural Networks, The First International Conference on Intelligent Computing in Data Sciences, Elsevier, 2018, pp.109-113. https://doi.org/10. 1016/j.procs.2018.01.104

[12] Yuchong Gu1, Yaoming Lai2, Peiliang Xie2, Jun Wei2, Yao Lu, Multi-Scale Prediction Network for Lung Segmentation, 16th International Symposium on Biomedical Imaging, IEEE, 2019, pp. 438-442.

[13] Tao Peng1, Yihuai Wang1, Thomas Canhao Xu1, (Member, IEEE), and Xinjian Chen, Segmentation of Lung in Chest Radiographs Using Hull and Closed Polygonal Line Method, IEEE Access, 2019, pp. 1-18. https://doi.org/10.1109/access.2019.2941511

[14] Armato SG, McLennan G, Bidaut L, McNitt-Gray MF, Meyer CR, Reeves AP, et al. H. Liu, et al. Physica Medica 63 (2019) 112-121, the lung image database consortium (lidc) and 
image database resource initiative (idri): a completed reference database of lung nodules on CT scans. Med Phys 2011; 38:915-31. https://doi.org/10.1118/1.3469350

\section{$6 \quad$ Authors}

Hayder Ayad currently works at the College of Business Administration, Al-Bayan University. Their current project is 'Visual object categorization'. He received his PhD. in computer science from university of technology, Iraq, 2017.

Ikhlas Watan Ghindawi is a lecturer at Collage of education, Computer science department, Al-Mustansiriyah University. Email: ikhlas watan@uomustansiriyah. edu.iq

Dr. Mustafa Salam Kadhm is an associate dean at faculty of Information Technology, Imam Ja'afar Al-Sadiq University. Email: muit.salam@sadiq.edu.iq

Article submitted 2020-07-17. Resubmitted 2020-10-11. Final acceptance 2020-10-13. Final version published as submitted by the authors. 